\title{
Diagnóstico de plantaciones comerciales de Tectona grandis L. f. en el trópico seco de Michoacán
}

\section{Diagnosis of commercial plantations of Tectona grandis L. f. in the dry tropics of Michoacan}

\section{H. Jesús Muñoz-Flores: \\ J. Trinidad Sáenz-Reyes 1 (1) \\ Rubén Barrera-Ramírez ${ }^{2 *}$ (]) \\ Jonathan Hernández-Ramos ${ }^{3}[0$ \\ Martín Gómez-Cárdenas: []}

${ }^{1}$ Instituto Nacional de Investigaciones Forestales Agrícolas

y Pecuarias (INIFAP)-CIRPACCampo Experimental Uruapan.

Av. Latinoamericana No.1110 Col.

Revolución, CP. 60150. Uruapan,

Michoacán, México.

${ }^{2}$ Universidad Autónoma de

Nuevo León-Facultad de Cien-

cias Forestales. Kilómetro 145

Carretera Nacional Cd. Victoria,

CP. 67700. Linares, Nuevo León,

México.

${ }^{3}$ INIFAP-CIRSE-Campo Experimental Chetumal, kilómetro 25 carretera Chetumal-Bacalar, CP. 77900. Othón P. Blanco. Quintana roo, México.

*Autor de correspondencia: ruben.barrera.ram@gmail.com

\section{Nota científica}

Recibida: 21 de junio 2021

Aceptada: 22 de octubre 2021

Como citar: Muñoz-Flores HJ, Sáenz-Reyes JT, BarreraRamírez R, Hernández-Ramos J, Gómez-Cárdenas M (2021) Diagnóstico de plantaciones comerciales de Tectona grandis L. F. en el trópico seco de Michoacán. Ecosistemas y Recursos Agropecuarios 8(3): e3058. DOI: 10.19136/era.a8n3.3058
RESUMEN. Para mitigar la deforestación en México, se han establecido plantaciones forestales comerciales (PFC) con especies nativas e introducidas. El objetivo fue realizar el diagnóstico del crecimiento, incremento y las características del suelo de plantaciones comerciales de teca (Tectona grandis L. f.), establecidas en el trópico seco del estado de Michoacán. El tipo de muestreo fue sistemático con intensidad entre 2.6 y $5.0 \%$. El análisis de varianza (ANOVA) indicó diferencias significativas en la supervivencia, crecimientos e incrementos y en área de copa, los más altos valores de supervivencia se tienen en la plantación San Bernardo (Municipio Nuevo Urecho) a siete años de su establecimiento, y los mayores incrementos medios anuales en diámetro, altura, volumen y área de copa, se presentaron en Las Casitas (Municipio de Múgica) a tres años. Para el estado fitosanitario y vigor del arbolado no se encontraron diferencias significativas.

Palabras clave: Teca, supervivencia, diámetro normal, incremento medio anual, propiedades del suelo.

ABSTRACT. To mitigate deforestation in Mexico, commercial forest plantations (FCP) with native and introduced species have been established. The objective was to diagnose the growth, increase and soil of commercial plantations of the introduced species teak (Tectona grandis L. f.), established in the dry tropics of the state of Michoacán. The type of sampling was systematic with intensity between 2.6 and $5.0 \%$. Analysis of variance (ANOVA) indicated significant differences in survival, growth and increments and in the crown, the highest survival values are found in the San Bernardo plantation (Nuevo Urecho municipality) seven years after its establishment, and the largest annual average increments in diameter, height, volume, and cup area, were presented in Las Casitas (Mugica municipality) at three years of age, and no significant differences were found in the plant health status and vigor of the tree.

Key words: Teca, survival, normal diameter, average annual increase, soil properties. 


\section{INTRODUCCIÓN}

En 2018, México perdió más de 267000 hectáreas (López y Mondragón 2019) por lo que un gran reto es detener los procesos de deforestación y degradación de suelos, mediante mejores prácticas de manejo forestal y el establecimiento de plantaciones forestales comerciales (PFC) (CONAFOR 2014). La superficie de PFC sigue siendo reducida y en el mismo año se plantaron 349.2 mil ha, de las cuales 222.9 mil ha corresponden a maderables, 101.3 mil ha a no maderables y 24.9 mil hectáreas de doble propósito (SNIGF 2019). Con más de 269 600 ha plantadas en el país, los estados de Veracruz, Tabasco, Campeche, Chiapas y Puebla son líderes al concentrar poco más del $65 \%$ del total (CONAFOR 2014).

La especie introducida T. grandis se encuentra entre las cinco principales especies, con la que se han plantado 30775 ha de PFC, hasta el 2018 (SNIGF 2019). Es una especie latifoliada que pertenece a la familia Verbenaceae y que puede alcanzar más de $50 \mathrm{~m}$ de altura y $2 \mathrm{~m}$ de diámetro en su lugar de origen, Indochina (Martínez-Zurimendi et al. 2015). Se adapta a una gran variedad de suelos, pero prefiere los aluviales de textura franco-arenoso 0 arcillosa, profundos, fértiles, bien drenados, $\mathrm{pH}$ neutro o ácidos y terrenos planos. Ha sido introducida en sitios con clima tropical, precipitación media anual entre 1500 y $2500 \mathrm{~mm}$, temperatura media entre 24 y $28^{\circ} \mathrm{C}$, suelos de textura media a moderadamente fina, profundos, bien drenados, con pH superior a 5.2 y en altitudes inferiores a $300 \mathrm{msnm}$ (Fonseca 2004, Martínez-Zurimendi et al. 2015).

En México, la madera tiene un alto valor económico y es empleada en mueblería, componentes decorativos y construcciones navales, puentes, durmientes de ferrocarril, pisos, construcción de muelles, instrumentos musicales, juguetes y barriles (Fonseca 2004, Ypushima et al. 2014).

En Cuba se reporta que plantaciones de cinco y 13 años, tuvieron diámetro promedio de 8 y $18.8 \mathrm{~cm}$, altura promedio de 5 y $7 \mathrm{~m}$, incremento medio anual en altura de 0.96 y $0.70 \mathrm{~m}$ año ${ }^{-1}$, y en volumen de
2.13 y $6.92 \mathrm{~m}^{3}$ ha año, además de incremento medio anual en diámetro de $1.6 \mathrm{~cm}$ año ${ }^{-1}$, en ambas plantaciones (González 2017). Al respecto, en Colombia se reporta que plantaciones de teca almacenaron entre 18.6 a $64.4 \mathrm{t}^{-1} \mathrm{C} \mathrm{ha}^{-1}$ con una tasa de fijación de carbono promedio de $1.4 \mathrm{t}^{-1} \mathrm{C}$ ha ${ }^{-1}$ año $^{-1}$ (Patiño et al. 2018).

En evaluaciones de plantaciones de T. grandis, Acrocarpus fraxinifolius y G. arborea en Michoacán, los resultados muestran que la más sobresaliente es la última especie, con $6.82 \mathrm{~m}$ de altura, DAP de 10.81 cm y $94.96 \%$ de supervivencia (Muñoz et al. 2009). Mientras que, en plantaciones de teca en Campeche, México, se desarrolló un sistema de crecimiento y rendimiento maderable de 152.75 y $136.86 \mathrm{~m}^{3}$ ha, respectivamente (Tamarit et al. 2019). En tanto que en la región centro-occidente de México, se elaboraron tablas de volúmenes para plantaciones de $T$. grandis con el modelo de variable combinada (Rueda et al. 2020).

La evaluación de las plantaciones forestales es una necesidad para conocer el crecimiento y rendimiento de las mismas y proponer su manejo silvícola. Sin embargo, en México y específicamente en Michoacán, existe escaza información sobre el comportamiento de $T$. grandis a las diferentes condiciones edafoclimáticas, lo que hace necesario la generación de conocimientos, principalmente en climas subtropicales (Muñoz et al. 2009). Por lo anterior, el objetivo del presente estudio fue realizar el diagnóstico del crecimiento, incremento y del suelo, de plantaciones comerciales de la especie introducida teca (Tectona grandis L. f.), establecidas en el trópico seco del estado de Michoacán.

\section{MATERIALES Y MÉTODOS}

\section{Área de estudio}

Las PFC evaluadas se ubican en los municipios de Nuevo Urecho y Múgica, que forman parte del trópico seco del estado de Michoacán (Tabla 1), las cuales recibieron financiamiento del Programa de Plantaciones Forestales Comerciales.

Dos de las PFC están establecidas en el municipio de Nuevo Urecho (San Bernando y El Mi- 
Tabla 1. Ubicación geográfica y condiciones edafoclimáticas de plantaciones forestales comerciales de Tectona grandis, en el trópico seco de Michoacán.

\begin{tabular}{|c|c|c|c|c|}
\hline Características/Predio & "El Mirador" & "San Bernardo" & "Las Casitas" & "El Tejón" \\
\hline Municipio & Nuevo Urecho & Nuevo Urecho & Múgica & Múgica \\
\hline Ubicación & $19^{\circ} 11^{\prime} 39.6^{\prime \prime} \mathrm{N}$ & $19^{\circ} 11^{\prime} 23.9^{\prime} \mathrm{N}^{\prime}$ & $18^{\circ} 56^{\prime} 31.4^{\prime \prime} \mathrm{N}$ & $18^{\circ} 56^{\prime} 11.4^{\prime \prime} \mathrm{N}$ \\
\hline geográfica & $101^{\circ} 51^{\prime} 53.3^{\prime \prime} \mathrm{W}$ & $101^{\circ} 53^{\prime} 55.8^{\prime \prime} \mathrm{W}$ & $102^{\circ} 05^{\prime} 11.0^{\prime \prime} \mathrm{W}$ & $102^{\circ} 08^{\prime} 58.8^{\prime \prime} \mathrm{W}$ \\
\hline Altitud (msnm) & 617 & 474 & 267 & 294 \\
\hline Edad (Años) & 7 & 7 & 3 & 4 \\
\hline Superficie (ha) & 3.00 & 3.85 & 7.40 & 8.94 \\
\hline Exposición & SW & Zenital & Zenital & Zenital \\
\hline \multirow[t]{2}{*}{ Clima } & Awo $(w)$ & Awo(w) & BSo(h')w(w) & BSo(h')w(w) \\
\hline & $\begin{array}{l}\text { Cálido subhúmedo } \\
\text { (El menos húmedo) }\end{array}$ & $\begin{array}{l}\text { Cálido subhúmedo } \\
\text { (El menos húmedo) }\end{array}$ & $\begin{array}{l}\text { Cálido seco } \\
\text { (Muy cálido) }\end{array}$ & $\begin{array}{l}\text { Cálido seco } \\
\text { (Muy cálido) }\end{array}$ \\
\hline Temperatura media anual $\left({ }^{\circ} \mathrm{C}\right)$ & 27 & 28 & 29 & 29 \\
\hline Precipitación media anual (mm) & 851 & 847 & 676 & 676 \\
\hline Pendiente & 2 & 1 & 1 & 1 \\
\hline Tipo de Suelo & Vertisol crómico & Vertisol crómico & Vertisol pélico & Vertisol pélico \\
\hline
\end{tabular}

rador), con densidades de 1667 árboles ha ${ }^{-1}$, y aplicación de riegos de auxilio durante la temporada de estiaje, así como deshierbes dos veces por año, durante los primeros tres años de su establecimiento. Las otras dos PFC se localizan en el municipio de Múgica (Las Casitas y El Tejón) con 1250 y 1000 árboles $\mathrm{ha}^{-1}$, respectivamente, a las que se les aplicó riego rodado cada 15 días durante el primer año y posteriormente una vez por mes durante los años siguientes. Para el control de maleza se aplicó tres veces por año, Faena ${ }^{\circledR}$ en dosis de $10 \mathrm{~mL}$ en un $L$ de agua y $20 \mathrm{~mL}$ de Coloso ${ }^{\circledR}$ en un $L$ de agua, durante los primeros tres años, además de barbechos cada año, y podas los primeros tres años desde su establecimiento.

\section{Muestreo}

Se utilizó un muestreo sistemático con sitios circulares de $250 \mathrm{~m}^{2}$ (radio $=8.92 \mathrm{~m}$ ) con intensidad de muestreo de 2.6 a $5.0 \%$, lo que representa un tamaño de muestra adecuado de acuerdo con Murillo et al. (2003).

Las variables cuantitativas evaluadas fueron: Supervivencia (S) a través de conteo directo de árboles vivos, diámetro normal (DN) a $1.30 \mathrm{~m}$, medido con cinta diamétrica marca Forestry Suppliers Inc. ${ }^{\circledR}$, altura total (AT) con un altímetro marca Haga ${ }^{\circledR}$, volumen del árbol y por superficie (V), y el área de copa (AC) se midió en los cuatro puntos cardinales, a partir del centro del fuste hacia la línea vertical imaginaria donde termina el follaje principal de la copa, para ello se empleó un longímetro marca Stanley ${ }^{\circledR}$ y posteriormente en gabinete se calculó el área de copa.

Para el cálculo del volumen de los árboles se utilizó la fórmula: $V=\left(D^{2}\right)(h)(0.7854)(0.6)$. Donde: $\mathrm{V}=$ Volumen $\mathrm{m}^{3}, \mathrm{D}=$ Diámetro normal $(\mathrm{m}), \mathrm{H}=$ Altura total $(\mathrm{m}), 0.7854=$ Factor resultante de dividir $\pi / 4$, de la fórmula del círculo y 0.6 = Coeficiente mórfico.

El volumen por hectárea en cada plantación, se estimó con el volumen promedio por árbol y la densidad actual en cada una de ellas. Los valores de incrementos medios anuales en diámetro normal (IMAD), altura total (IMAA) y volumen (IMAV), se determinaron entre los resultados obtenidos en las variables entre la edad de las plantaciones.

Para calcular el área de copa se utilizó la fórmula de la elipse: $A=\left(D_{1}\right)\left(D_{2}\right)$ (0.7854). Donde: $A=$ Área de copa $\left(\mathrm{m}^{2}\right), \mathrm{D}_{1}=$ Diámetro medio de Norte a Sur $(m)$ y $D_{2}=$ Diámetro medio de Este a Oeste $(m)$.

Además, se evaluaron las siguientes variables cualitativas: a) Estado fitosanitario. Se consideraron dos conceptos: sano o enfermo, se determinó como árbol enfermo cuando el daño es evidente, además, que tenga un posible efecto con el crecimiento, y b) Vigor. Se refiere a las características fenotípicas del árbol y se clasificaron en: exuberantes: aquellos que sobresalieron de la media de la población, normales: árboles que se encontraron dentro de la media y raquíticos: árboles de la misma edad pero que presentan un desarrollo muy deficiente.

Se tomaron dos muestras de suelo para cono- 
cer sus propiedades físico-química y los resultados se compararon con las especificaciones de la NOM021-RECNAT-2000 (SEMARNAT 2002) y con estudios realizados en PFC a nivel mundial. Para cada variable evaluada se realizaron análisis de varianza (ANOVA) y comparación de medias de Tukey $(\mathrm{P}<$ $0.05)$, con el lenguaje de programación $R$ versión 3.4.3 (R Core Team 2017), con un nivel de confianza del $95 \%$.

\section{RESULTADOS Y DISCUSIÓN}

\section{Variables cuantitativas y cualitativas}

En las variables cuantitativas, el ANOVA indica diferencias significativas $(P<0.05)$ en DN, IMAD, AT, IMAA, V e IMAV, presentando los mayores valores de crecimiento las PFC establecidas en El Mirador y San Bernardo de siete años; pero los mayores valores en IMAD e IMAA se presentaron en la PFC Las Casitas, con tres años y en el IMAV en las PFC San Bernardo y Las Casitas (Figura 1). Para la supervivencia se encontraron diferencias significativas $(P<0.05)$, presentando los mayores valores en las PFC San Bernardo, Las Casitas y El Mirador (Figura 1). Los mayores valores de DN se deben principalmente a que las plantaciones son de mayor edad y al manejo post plantación (aplicación de riego, podas y deshierbes), los cuales son similares a los reportados en PFC de T. grandis a los seis años $(14.0 \mathrm{~cm})$ en el predio $\mathrm{El}$ Mirador 2 en Nayarit (Salcedo et al. 2019), así como a las evaluadas a los siete años $(15.3$ y $19.9 \mathrm{~cm}$ ) en Costa Rica (Moya et al. 2010).

Los valores altos de IMAD se tienen en las PFC más jóvenes y conforme incrementa la edad disminuye su incremento. Los IMAD estimados en las plantaciones del municipio de Múgica son superiores en comparación a los determinados en PFC de $T$. grandis a los seis años $\left(2.2 \mathrm{~cm}\right.$ año $\left.{ }^{-1}\right)$ en El Mirador 2 en Nayarit, con clima cálido subhúmedo con lluvias en verano, altitud de $25 \mathrm{~m}$, precipitación anual de 1 $496 \mathrm{~mm}$ y temperatura promedio anual de $23.5^{\circ} \mathrm{C}$ (Salcedo et al. 2019).

Las alturas de los árboles en las plantaciones evaluadas son inferiores a las logradas en PFC de
T. grandis a los seis años $(12.0 \mathrm{~m})$ en Nayarit (Salcedo et al. 2019), y a lo reportado en plantaciones en el norte y noroeste ( 14.9 y $18.1 \mathrm{~m}$ ) de Costa Rica, a la misma edad de siete años, con densidades de plantación de 396 y 377 árboles ha $^{-1}$ (Moya et al. 2010). Los IMAA de las dos PFC del municipio de Nuevo Urecho son inferiores a lo reportado en plantaciones a los seis años $\left(2.3 \mathrm{~m}_{\text {ñ̃o }}{ }^{-1}\right)$ en Nayarit (Salcedo et al. 2019) y solamente superado por la PFC Las Casitas con $3.3 \mathrm{~m}_{\text {año }}{ }^{-1}$ (Figura 1). Los resultados en este estudio indican que los mayores valores de IMAA se tienen en las PFC más jóvenes y conforme incrementa la edad disminuye su incremento.

Los valores más altos en volumen por hectárea se presentaron en las PFC del municipio Nuevo Urecho, con 126.1 y $102.8 \mathrm{~m}^{3} \mathrm{ha}^{-1}$ (Figura 1C), lo cual se debe principalmente a la edad, al crecimiento en DN y altura que presentaron y la mayor densidad con 1467 y 1600 árboles ha $^{-1}$ y son superiores a los citados por CONAFOR (2013) de $89.2 \mathrm{~m}^{3} \mathrm{ha}^{-1}$ y a la de $T$. grandis a los seis años $\left(98 \mathrm{~m}^{3} \mathrm{ha}^{-1}\right)$ en Nayarit (Salcedo et al. 2019). Los mayores valores de IMAV, estimados en Las Casitas y San Bernardo, son superiores a los registrados en San Blas, Nayarit (16.9 $\mathrm{m}^{3}$ ha $^{-1}$ año $^{-1}$ ) con clima tropical seco (Ypushima et al. 2014), al igual que a las PFC de T. grandis establecidas en los municipios de Villa de Álvarez y Comala, Colima, donde el IMAV fue de 2.1 y $2.7 \mathrm{~m}^{3}$ ha $^{-1}$ año $^{-1}$, respectivamente (Muñoz et al. 2011) y a las ubicadas en el Canal de Panamá $\left(2.9^{-1} 3.3\right.$ $\mathrm{m}^{3} \mathrm{ha}^{-1}$ año $^{-1}$ ) (Mollinedo et al. 2005). Los resultados en $S$ coinciden a los reportados por Muñoz et al. (2009), en el municipio de Nuevo Urecho, Mich., donde $T$. grandis logró una supervivencia de $86.32 \%$ a los dos años.

En las variables cualitativas, los ANOVA's indican que se encontraron diferencias significativas $(P$ $<0.05$ ) en AC, donde los mayores valores se reportan en las PFC de los predios Las Casitas y San Bernardo y en VI y EF no se encontraron diferencias significativas $(p<0.05)$ y su rango fue del 90 a $100 \%$ de árboles vigorosos (Tabla 2).

El mayor valor de AC correspondió a la PFC establecida en el paraje Las Casitas con $6.85 \mathrm{~m}^{2}$, mientras que la menor $\left(1.95 \mathrm{~m}^{2}\right)$ se presentó en el 

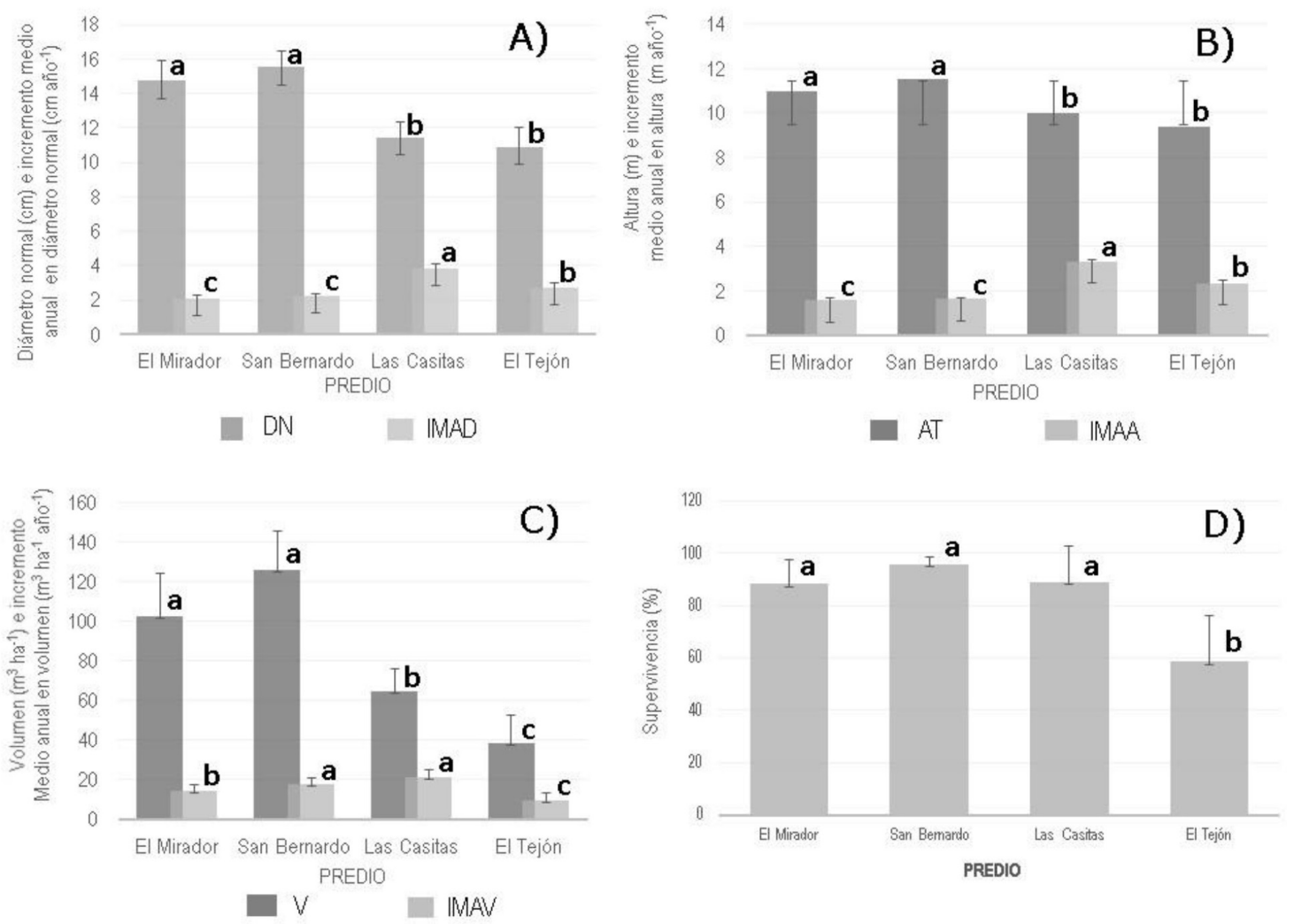

Figura 1. Comparación de medias de las plantaciones comerciales de Tectona grandis, en el trópico seco de Michoacán. A). Diámetro normal e incremento medio anual en diámetro. B). Altura total e incremento medio anual en altura. C). Volumen e incremento medio anual en volumen. D). Supervivencia. Donde: Medias con distintas letras son estadísticamente diferentes (Tukey, $\mathrm{P}<$ 0.05). Las líneas sobre las barras indican la desviación estándar.

Tabla 2. Comparación de medias de variables cualitativas de plantaciones comerciales de Tectona grandis, en el trópico seco de Michoacán.

\begin{tabular}{lccc}
\hline $\begin{array}{l}\text { Variables } \\
\text { /Paraje }\end{array}$ & $\begin{array}{c}\text { Vigor } \\
(\%)\end{array}$ & $\begin{array}{c}\text { Área de copa } \\
\left(\mathrm{m}^{2}\right)\end{array}$ & $\begin{array}{c}\text { Estado fitosanitario (Sin daños) } \\
(\%)\end{array}$ \\
\hline El Mirador & $90(25.1) \mathrm{a}$ & $1.95(0.7) \mathrm{c}$ & $100(0.0) \mathrm{a}$ \\
S. Bernardo & $100(0.0) \mathrm{a}$ & $6.28(1.2) \mathrm{a}$ & $100(0.0) \mathrm{a}$ \\
Las Casitas & $100(0.0) \mathrm{a}$ & $6.85(1.8) \mathrm{a}$ & $100(0.0) \mathrm{a}$ \\
El Tejón & $100(0.0) \mathrm{a}$ & $4.26(1.0) \mathrm{b}$ & $100(0.0) \mathrm{a}$ \\
\hline
\end{tabular}

Donde: Cantidades entre paréntesis corresponden a la desviación estándar. Medias con distintas letras son estadísticamente diferentes (Tukey: $P<0.05$ ).

paraje El Mirador (Tabla 2). La variación del AC se debe a las condiciones actuales que guarda cada una de ellas, en cuanto a la densidad de arbolado y a la competencia por luz, nutrimentos, humedad y espacio, junto con las labores post plantación que han realizado como riegos, podas y aclareos.

\section{Tipo de suelo y características físico-químicas}

En la Tabla 3 se muestran los resultados de los análisis de suelo. El tipo de suelo es vertisol y de textura arcillosa, que no son los más adecuados para el establecimiento de PFC de T. grandis, ya que difiere con lo señalado por Salcedo et al. (2019). Asimismo, el $\mathrm{pH}(7.6)$ y el porcentaje de $\mathrm{MO}(0.54 \%)$ de la PFC Las Casitas, así la MO de El Mirador (1.29\%), no están dentro de los valores ( $\mathrm{pH}$ 6.0-7.0 y $\mathrm{MO}>2.5 \%$ ) citados por Salcedo et al. (2019), con los cuales se obtuvieron los mayores incrementos y productividad 
Tabla 3. Resultados de los análisis de suelo de plantaciones forestales comerciales de Tectona grandis, en el trópico seco de Michoacán.

\begin{tabular}{lcc}
\hline \multirow{2}{*}{$\begin{array}{l}\text { Sitio/ } \\
\text { Variable }\end{array}$} & $\begin{array}{c}\text { "El Mirador", } \\
\text { Mpio. Nvo. Urecho, Mich. }\end{array}$ & $\begin{array}{c}\text { "Las Casitas", } \\
\text { Mpio. Múgica, Mich. }\end{array}$ \\
\cline { 2 - 3 } pH agua (1:2) & \multicolumn{2}{c}{ Resultados } \\
Materia orgánica & 6.57 & 7.60 \\
Textura & $1.29 \%$ & $0.54 \%$ \\
Arena & Arcillosa & Arcillosa \\
Limo & $18.40 \%$ & $18.04 \%$ \\
Arcilla & $28.00 \%$ & $20.36 \%$ \\
Nitrógeno (N asimilable) & $53.60 \%$ & $61.60 \%$ \\
Fósforo (P)total & $137.09 \mathrm{~kg} \mathrm{ha}^{-1}$ & $140.42 \mathrm{~kg} \mathrm{ha}^{-1}$ \\
Potasio (K) & $16.58 \mathrm{~kg} \mathrm{ha}^{-1}$ & $13.83 \mathrm{~kg} \mathrm{ha}{ }^{-1}$ \\
Azufre (S) & $244.17 \mathrm{~kg} \mathrm{ha}^{-1}$ & $205.01 \mathrm{~kg} \mathrm{ha}$ \\
Calcio (Ca) & $1.43 \mathrm{ppm}$ & $1.57 \mathrm{ppm}$ \\
Magnesio (Mg) & $765.71 \mathrm{ppm}$ & $987.10 \mathrm{ppm}$ \\
Boro (B) & $79.86 \mathrm{ppm}$ & $109.46 \mathrm{ppm}$ \\
Hierro (Fe) & $0.47 \mathrm{ppm}$ & $0.57 \mathrm{ppm}$ \\
Zinc (Zn) & $44.22 \mathrm{ppm}$ & $41.80 \mathrm{ppm}$ \\
Cobre (Cu) & $0.53 \mathrm{ppm}$ & $0.49 \mathrm{ppm}$ \\
Manganeso (Mn) & $1.21 \mathrm{ppm}$ & $1.03 \mathrm{ppm}$ \\
Sodio (Na) & $2.30 \mathrm{ppm}$ & $2.14 \mathrm{ppm}$ \\
Aluminio (Al) & $42.56 \mathrm{ppm}$ & $58.07 \mathrm{ppm}$ \\
\hline
\end{tabular}

en PFC de T. grandis, a la edad de seis años en $\mathrm{Na}$ yarit.

Los mayores valores de diámetros (15.52 y $14.72 \mathrm{~cm}$ ), altura total (11.57 y $10.97 \mathrm{~m}$ ) y volumen $\left(126.13\right.$ y $\left.102.78 \mathrm{~m}^{3}\right)$ se registraron en las PFC del municipio de Nuevo Urecho, a los siete años de edad; pero, los mejores incrementos medios anuales (3.81 $\mathrm{cm}, 3.33 \mathrm{~m}$ y $21.64 \mathrm{~m}^{3} \mathrm{ha}^{-1} \mathrm{año}^{-1}$ ) se presentaron en la plantación Las Casitas con edad de tres años. El estado fitosanitario es sano y el vigor del arbolado fue bueno. La caracterización físico-química del suelo mostró deficiencia de macro y micronutrientes, que se ve reflejada en bajos crecimientos e incrementos de las plantaciones, principalmente en el suelo vertisol crómico.

\section{LITERATURA CITADA}

CONAFOR (2013) Programa de desarrollo de plantaciones forestales comerciales, a 15 años de su creación. Comisión Nacional Forestal-Colegio de Postgraduados. México, México. 472p.

CONAFOR (2014) México cuenta con 270 mil hectáreas de Plantaciones Forestales Comerciales. Unidad de Comunicación Social. Boletín 77. Secretaría de Medio Ambiente y Recursos Naturales. México. 2p.

Fonseca GW (2004) Manual para productores de teca (Tectona grandis L.f.) en Costa Rica. Heredia, Costa Rica. $115 p$.

González BE (2017) Evaluación del crecimiento de las plantaciones de Tectona grandis L.F. Unidad silvícola Mayarí. Revista Cubana de Ciencia Forestales 5: 330-339.

López PV, Mondragón M (2019) GFW Technology Offers Options to Combat Mexico's High Rate of Tree Cover Loss. Version 10 G Street NE Suite 800, Washington, DC 20002, USA. https://blog.globalforestwatch.org/ data-and-research/gfw-technology-offers-options-to-combat-mexicos-high-rate-of-tree-cover-loss. Fecha de consulta: 15 de febrero de 2021.

Martínez-Zurimendi P, Domínguez-Domínguez M, Juárez-García A, López-López LM, De la Cruz-Arias V, ÁlvarezMartínez J (2015) Índice de sitio y producción maderable en plantaciones forestales de Gmelina arborea en 
Tabasco, México. Revista Fitotecnia en México 38: 415-425.

Mollinedo M, Ugalde L, Alvarado A, Mark VJ, Carles RL (2005) Relación suelo-árbol y factores de sitio, en plantaciones jóvenes de teca (Tectona grandis), en la zona oeste de la cuenca del canal de Panamá. Agronomía Costarricense 29: 67-75.

Moya RL, Arce VL, González PE, Olivares GC, Ríos GV (2010) Efecto de las propiedades físicas y químicas del suelo en algunas propiedades de la madera de teca (Tectona grandis). Revista Árvore 34: 1109-1118.

Muñoz FHJ, Coria AVM, García SJJ, Balam MCh (2009) Evaluación de una plantación de tres especies tropicales de rápido crecimiento en Nuevo Urecho, Michoacán. Ciencia Forestal en México 34: 61-87.

Muñoz FHJ, Orozco GG, Sáenz RJT, Rueda SA, Prieto JAR (2011) Evaluación de plantaciones forestales comerciales tropicales en el estado de Colima y Michoacán. Folleto Técnico Núm. 25. SAGARPA. INIFAP. CIRPAC. Campo Experimental Uruapan. Michoacán, México. 71p.

Murillo GO, Badilla V, Gallegos RA (2003) Guía para la evaluación de la calidad del establecimiento de plantaciones forestales. Instituto Tecnológico de Costa Rica-Universidad de Guadalajara. Guadalajara, Jalisco, México. 37p.

Patiño FS, Suárez SLN, Andrade CHJ, Segura MMA (2018) Captura de carbono en biomasa en plantaciones forestales y sistemas agroforestales en Armero-Guayabal, Tolima, Colombia. Revista De Investigación Agraria y Ambiental 9: 121-134.

R Core Team (2017) R project 4.3.4. https://www.r-project.org/. Fecha de consulta: 16 de febrero de 2019.

Rueda SA, Benavides SJD, García GDA, Sáenz RJT, Castillo QD, García MGE (2020) Tablas de volumen para Tectona grandis L.f. en plantaciones del centro-occidente de México. Revista Latinoamericana de Recursos Naturales 16: 96-102.

Salcedo PE, Ruíz BBA, Hernández ÁE, González CR, Bernabé AA, Orozco GE, Ramírez LCB, Anzaldo HJA, Delgado FE (2019) Propiedades del suelo y nitrógeno como indicadores del crecimiento en plantaciones comerciales de teca. Revista Mexicana de Ciencias Forestales 10: 33-54.

SEMARNAT (2002) Norma Oficial Mexicana NOM-021-RECNAT-2000, que establece las especificaciones de fertilidad, salinidad y clasificación de suelos, estudio, muestreo y análisis. Diario oficial. Secretaría del Medio Ambiente y Recursos Naturales. http://www.ordenjuridico.gob.mx/Documentos/Federal/wo69255.pdf. Fecha de consulta: 23 de marzo de 2021.

SNIGF (2019) Evaluaciones de plantaciones forestales comerciales y reforestación con propósitos de restauración y conservación. Sistema Nacional de Investigación y Gestión Forestal. Informe final. 147p. https:// snigf.cnf.gob.mx/evaluaciones. Fecha de consulta: 22 de enero de 2021.

Tamarit UJC, De los Santos P, Aldrete AHM, Valdez LJR, Ramírez MH, Guerra de la CV (2019) Sistema de crecimiento y rendimiento maderable para plantaciones de teca (Tectona grandis L.f.) en Campeche, México. Madera y Bosques 25: 1-16.

Ypushima PAL, Salcedo PE, Manríquez GR, Silva GJA, Zamora NJF, Hernández ÁE (2014) Propiedades de la madera y relación del estado nutrimental con el crecimiento en teca. Revista Mexicana de Ciencias Forestales 5: 26-39. 